\title{
DISTRIBUTION ISSUES IN THE DESIGN AND IMPLEMENTATION OF A VIRTUAL MARKET PLACE
}

\author{
Yigal Hoffner, Christian Facciorusso, \\ Simon Field and Andreas Schade
}

\author{
IBM Research Division, Zürich Research Laboratory \\ Säumerstrasse 4, CH-8803 Rüschlikon, Switzerland \\ yho|cfa|sif|san @ zurich.ibm.com
}

\begin{abstract}
This paper describes an investigation into the distribution issues surrounding the design and implementation of virtual market places. The paper starts by describing the requirements customers and service providers have from a virtual market place. It is then shown how the requirements deemed most important can be addressed by exploiting the inherent distribution of certain aspects, and by distributing other aspects of such market places. In particular, the paper concentrates on the structuring of the information space describing the services and products, and its distribution between the providers and the market place mediator. Primarily, this supports the implementation of phased dialogues between the customer and the providers, while at the same time addressing the providers' desire to protect business sensitive processes and information. Distributing the dialogue in time allows customers and providers to interact more flexibly and to gradually build understanding and trust between them, before divulging any business or personally sensitive information to each other.

The distributed approach supports a scalable market place and also points towards a way of addressing providers' legacy problems. It is also shown how the apparent contradiction of a distributed market place and the need to monitor and control it for regulation purposes can be addressed, without compromising the ability of the system to scale.

Finally, the design and implementation of a virtual insurance market place using Java on a CORBA compliant platform are presented.
\end{abstract}

Keywords: Electronic market places, virtual market middleware, dialogues, market place information (advertising and searching) space, match-making, CORBA trading service 


\section{INTRODUCTION}

Designers facing the task of designing and implementing virtual market places must deal with complex, varied and sometimes conflicting factors and requirements, operate within the constraints of environments which are not entirely under their control, and deal with market place rules and regulations which further complicate their task [6] [14] [2] [3].

The mixture of concerns, which has to be covered, coupled with currently evolving technology, offers the designers a large spectrum of options concerning how to design and implement such systems. How is a designer then to tackle the task?

This paper proposes an approach, which is based on a careful analysis of the requirements which customers and providers have from a market place. The approach suggests exploiting the distribution inherent in such market places where possible, and introducing and enhancing it where it may not already exist. The distributed approach addresses the market place requirements deemed most important and can be used to create a variety of systems, each of which can be tailored to the specific requirements and the particular flavour of a different market domain.

Chapter 2 describes the requirements which customers and providers have from virtual market places. Chapter 3 selects the most important requirements described in Chapter 2; these point towards the need to exploit the inherent distribution of certain aspects, and the need to distribute other aspects of such market places. The chapter outlines the principles upon which our distributed market place architecture is based. The rest of the paper demonstrates how these principles are applied in the design of our Virtual Market Place (ViMP) system. Chapter 4 discusses the relationship between the logical structuring of the market-place information space which describes the market goods, and the manner in which this information can be physically distributed in the market place. The chapter then proceeds to show how to structure and distribute the dialogues between the customer and the market place. Chapter 5 checks how the requirements not directly addressed by distribution or those that appear to contradict the distributed approach can be dealt with. Chapter 6 presents the first prototype of the market place (ViMP) based on the architecture described in this paper. Chapter 7 presents the conclusions drawn from the virtual market place architecture and its use in the design and implementation of a specific market place. Future avenues of research and work are then pointed out.

\section{VIRTUAL MARKET PLACE REQUIREMENTS}

This chapter outlines some basic market place terminology and then sets out the requirements which customers and providers have from virtual market places. These serve as the background and the motivation for the rest of the paper.

\subsection{Market place terminology}

The term goods, which is used throughout this paper describes both products and services which may be electronic or non-electronic in nature. It is therefore assumed that while browsing and shopping is done within the computer system, the goods 
may be delivered outside the computer system. This poses different requirements and constraints on a virtual market place in terms of monitoring and auditing, for example.

The term market place mediator describes the entity, which resides between the customer and the providers of the market place. In some market places the mediator may only provide a known point of entry to the market place, while in others it may have varying degrees of involvement in the ensuing dialogue between the customer and the providers.

\subsection{Customer requirements from a virtual market place}

As far as customers are concerned, electronic market places have to:

1. Support a recognised point of entry: Customers want an easy to find point of entry to the market place.

2. Provide choice for the customer: Customers would like the market place to give them access to a large number of provider companies (offering competition), and a wide variety of goods (offering choice), reducing their need to visit other locations in addition to the market place.

3. Create an audited and regulated market place: Customers like to know that their interests are being protected. An audited market place can help provide protection and offer a mechanism to resolve disputes. For some business areas (e.g. financial services and insurance), customer confidence in the market place will be improved if the presence of providers in the market place is regulated by a suitable authority.

4. Enable the customer to supply necessary information only once: Customers would prefer to specify what they want, and provide appropriate information once only, rather than have to fill multiple (and often similar) forms for each provider.

5. Facilitate goods selection - receive only relevant offers: Customers wish to have the ability to filter the large volume of offers available, and home onto the goods, which most closely match their requirements.

6. Protect confidential information: In some types of market, customers will be required to divulge information, which they will consider to be confidential. They would like assurances that the market place will treat such confidential information appropriately.

7. Enable the customer to provide confidential data only when necessary: Customers are reluctant to divulge personal or confidential information before any level of confidence has been established between them and the providers in the market place. Confidence in this context can come from a feeling that the providers have a reasonable understanding of what the customer wants, that they can actually offer something relevant, and that the information provided will be treated confidentially.

8. Enable the customer to explore offer feature space: Goods are often complex, with a variety of features and priced options. Customers wish to be able to 
explore the feature space of offers, so that they can compare the features of offered goods, and assess the trade-off between the price and available features of competing goods.

9. Support interruptible long-life sessions: Customers may wish to be able to suspend a dialogue before it has reached a conclusion, and resume it at a later stage.

10. Reward loyalty: Customers wish to be rewarded for purchasing multiple goods from one provider, either at the same time, or over a period of time.

11. Provide protection from direct access: Many customers do not wish to be bothered by unsolicited email, and would not wish their identity to be made available to all providers in the market place.

\subsection{Provider requirements from a virtual market place}

As far as provider organisations are concerned, electronic market places have to:

1. Provide a well-visited market place: Providers will be more inclined to join a market place if they believe that it will be visited by many potentially profitable customers. As customers are more likely to visit a market place populated by many providers, a consequence of this requirement is the willingness of providers to join market places alongside their competitors.

2. Support complete and dynamic control of the advertising: Providers wish to be able to change their presence in the market place dynamically. They may wish to update existing offers, revoke old offers or introduce new offers.

3. Protect commercially sensitive information and business processes: Preparing an offer for a customer may involve commercially sensitive information or business processes, which the provider will wish to keep within their organisational boundary, while allowing the market place access to them when necessary.

4. Allow integration and exploitation of legacy systems: Providers are likely to have information about their goods already stored in existing applications and databases. When providing offers via the market place, providers would wish to re-use such existing information rather than wastefully replicate it.

5. Create an audited and regulated market place: Reputable providers will wish to see that competition across the market place is fair, and that regulations are complied with. Auditing will help ensure that disputes are dealt with fairly.

6. Avoid unnecessary interaction with customers: Providers would like to target certain customer groups, to be applied to potential customers before any lengthy dialogue takes place. This will help to minimise the expense of handling customer enquiries via the market place.

7. Allow provider and goods differentiation and specialisation: Providers wish to be able to differentiate themselves from their competitors, by way of corporate branding and by highlighting the features of offered goods. 
8. Allow for human intervention in decision process: Providers may wish to involve their staff in the process of deciding whether to put one or more offers to a customer. The system must therefore allow a provider to take time in deciding how to respond to customer requests via the market place.

9. Provide special customer treatment: Providers may wish to tailor offers for individual customers. For example they may wish to treat customers differently according to their previous customer history, or provide incentives for customers buying multiple goods.

\section{THE APPROACH: DISTRIBUTING THE MARKET PLACE}

This chapter selects the most important requirements described in Chapter 2 and shows how they point towards the need to exploit and enhance the distribution aspects of virtual market places. The chapter also outlines the principles upon which our distributed market place architecture is based.

\subsection{Prioritising the market place requirements}

The design space of virtual market places is large, complex and driven by many varied and sometimes conflicting factors and requirements. Without some sense of priority in addressing these, it is possible to come up with a solution that partially addresses everybody's concerns while satisfying nobody. Therefore, out of the above customer and provider requirements list, we chose those requirements that were considered to have the highest priority for the following reasons:

- They dictate the structure and organisation of the information and the processes of the market place over space and time.

- They define the nature of the market place and its operational mode.

According to the above criteria, the prime requirements (summarised from 2.2 and 2.3) which should motivate the virtual market place architecture are:

- Only provide personal and confidential data when necessary.

- Avoid unnecessary interaction with customers.

- Allow integration and exploitation of legacy systems.

- Protect commercially sensitive information and business processes.

- Create an audited and regulated market place.

- Provide complete and dynamic control of the advertised goods. 


\subsection{Trends and questions in current virtual market places}

For reasons of trust, a market place which hosts competing providers will usually require a market place mediator to be a legally independent entity, and therefore often physically separate from the individual providers. Given this separation between the providers and the mediator, a central question arises concerning just how much of the overall market place will have to be delegated to that mediator. In other words, how much of the provider's information and processes will have to be moved to the market place itself?

One past trend in some early computerised market places was to require the participating providers to advertise all their goods in the mediator [8] [13]. This complicates the process of updating the information. Furthermore, the technology used to update the information influences the update turn around time, and must be compatible with the requirements of the dynamic markets currently envisioned [3]. Provider organisations traditionally have their business sensitive processes and information reside within their organisations. Replicating these processes outside the organisation relies on the willingness of the providers to supply business sensitive information to the market place, which in some cases includes algorithms and tables, which expose the pricing policy of the provider, for example. It may also be too difficult or expensive to do so for legacy reasons. In addition, it requires a uniform representation of the algorithms and the information so that they can be used by the mediator, making it a difficult and expensive requirement to fulfil.

Another trend in some existing electronic market places is to provide a single stage dialogue between the customer and the market place, in which the customer is presented with a single page-long questionnaire, which has to be completed before it can be sent to the market place. Such a form of interaction provides no meaningful feedback until the entire form has been filled, and assumes that customers have apriory understanding of all the questions asked and the implications of their choices. Providing intermediate feedback about the customer choices and answers would be a far better approach, particularly in markets where the goods are of a complex nature.

\subsection{Exploiting and creating distribution in the market place}

One way of addressing the high priority requirements described in 3.1 without making the same mistakes of some existing market places, is to exploit any inherent distribution which already exists, and to create further distribution of the virtual market place in space, time and representation. This can be achieved:

1. By distributing the market place information spatially inside and outside the provider's organisation rather than keeping it all in the mediator or within the provider's boundary:

The requirement to hand over most if not all of the market place information and processes to the mediator can be relaxed; the entire market place does not have to be separated from the providers and handed to the mediator. It is sufficient that the entry point and whatever preliminary selection process is agreed by the participants, takes place in the mediator. The rest of the interactions can take place with the providers, either directly or through the mediator. On the other hand, delegating more of the early match-making process to a mediator can filter 
potential customers and help satisfy the requirement of avoiding unnecessary interactions with unsuitable customers.

Distributing the market information rather than storing it centrally in the mediator makes the resulting market place more scalable.

2. By structuring the interactions between the customer and the mediator and distributing them in time, to create phased dialogues:

The phasing of the dialogue allows the provision of intermediate feedback to customers and ensures that they are not overwhelmed with page-long questionnaires. It also caters for the gradual build-up of understanding and trust between the customer and the market place.

The distribution of the market place between the mediator and the providers, coupled with the notion of phasing the dialogue, implies having the initial interaction(s) with the mediator and the subsequent ones with the providers. Only customers who fit the providers' initial criteria and whose requirements match to initial choices given by the providers, go to the next phases of the dialogue, which ultimately will provide access to the providers. This addresses the providers' requirement of avoiding unnecessary interaction with customers and the customer requirement to facilitate goods selection and receive only relevant offers.

However, the option of whether the dialogue continues to take place through the mediator or is then conducted directly between the customer and providers is a market place specific decision. Market places with a strong regulatory aspect may be best served by a tight (or 'centralised') dialogue control model, where the entire dialogue is conducted through the mediator, but where the mediator's role in later phases is that of a collator and distributor of information between the customer and providers. While in a more loose dialogue control model, the mediator would merely set-up the contact between the customer and the individual providers of mutual interest, and the rest of the dialogue would be conducted directly with them.

The distribution of the market place can be further exploited to facilitate multiple dialogues to be simultaneously conducted with several providers. This, however, raises the problem of managing multiple dialogues, as well as requiring a large degree of agreement between the market place providers about the dialogue structure and content.

3. By accepting the variety of representations and processes within provider organisations and their legacy systems:

A different approach to creating a single uniform way of carrying out certain processes and of representing information and of forcing the providers to change their organisations accordingly should be tried. Instead, it is possible to connect existing equipment and legacy systems by wrapping them with the chosen distributed application technology, and by translating the necessary information into the market place standard where necessary. This also caters for any interactions with autonomous organisations, which are outside the control of the market place. 
Some of the most important decisions facing a market place designer therefore concern how to exploit inherent distribution and where to introduce further distribution:

- How much of the goods, the provider information and the selection process should stay with the providers and how much of it should be delegated to the mediator?

- How should the dialogue be phased and what kind of feedback should the customer get at each phase of the dialogue?

- How to deal with the legacy systems in the market place and what distributed system technology to wrap them with, so that the market place can use them productively?

Many of the decisions of how exactly to distribute the market place will depend on its objectives, its specific nature, the type of providers and customers, etc., making it difficult to have a single solution which would fit every type of market place. However, a solid virtual market place architecture and development tools should provide the designer with a spectrum of options and make them aware of the choices, the trade-off between them and the implications of their decisions. Furthermore, the decisions concerning the distribution in space and time and representation are not orthogonal but dependent on each other, and where possible, the dependencies should be pointed out.

\section{THE DISTRIBUTION OF THE MARKET PLACE INFORMATION SPACE AND DIALOGUES}

This chapter describes an approach to structuring and distributing the information space - where the advertising and searching for compatible goods takes place in a virtual market place. Having set the context of the distribution of the market place information space, the chapter looks at the manner in which this can be exploited to support phased dialogues between the customer and the market place.

\subsection{Structuring the market place information space}

The exact structuring of the market place information space can be influenced by the requirements, which support the phasing of the dialogue. The exact needs of the dialogue will clearly depend on the type of the market, its goods and on the customer.

Nevertheless, some generalities can be outlined, for example, at the highest level, a company can describe its lines of goods in abstract terms and provides some information about the generic quality guarantees it is willing to give. This approach is aimed at creating a hierarchical information (advertising-searching) space as shown in Figure 1.

By structuring and dividing the description of market goods and similarly of the information requested from the customer at each phase, it is possible to stagger the advertising of the goods into the market place. This scheme enables a degree of 
distribution of the information space, since the more detailed and business sensitive information can be kept within the provider organisation, and the more high-level and less sensitive information can be sent to the mediator, who can itself be further distributed if necessary.

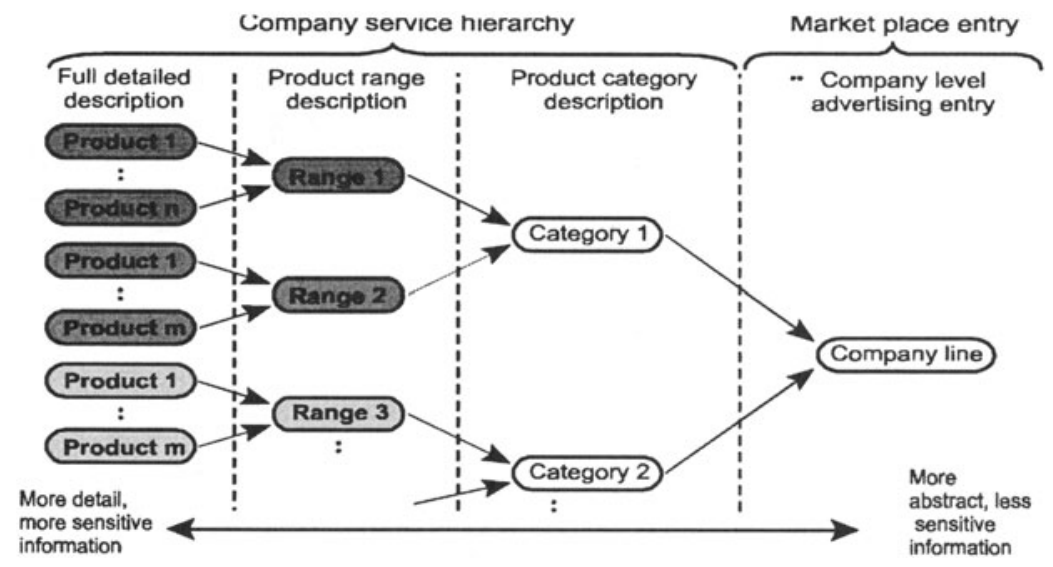

Figure 1. A hierarchical scheme for describing the goods of a service provider.

\subsection{Distributing the market place information space}

A match-making engine (MME) is a mechanism capable of receiving the description and requirements of a service or a product and of a customer, and matching the description of each against the requirement of the other. This topic is further discussed in 5.2.1 and in [7] and [5].

The hierarchical structuring of the information space shown in Figure 1 can be used to physically distribute the information space. One approach to implementing this type of configuration is to assign a match-making engine to each level of service description, but other schemes are also possible. This approach results in a hierarchical structure of match-making engines shown in Figure 2.

For the distribution scheme to succeed, the different MMEs of the market place, both outside and inside the providers, must know of each other and be able to communicate with each other or with the customer, so as to achieve a unified market place. Allowing some parts of the information and processes to stay within the organisational boundaries also addresses the problem of legacy systems. These can now be wrapped to provide the translation and communication necessary to connect them to the market place. Business sensitive information and processes can now be left inside the organisations and accessed when the need arises to provide the necessary information. 


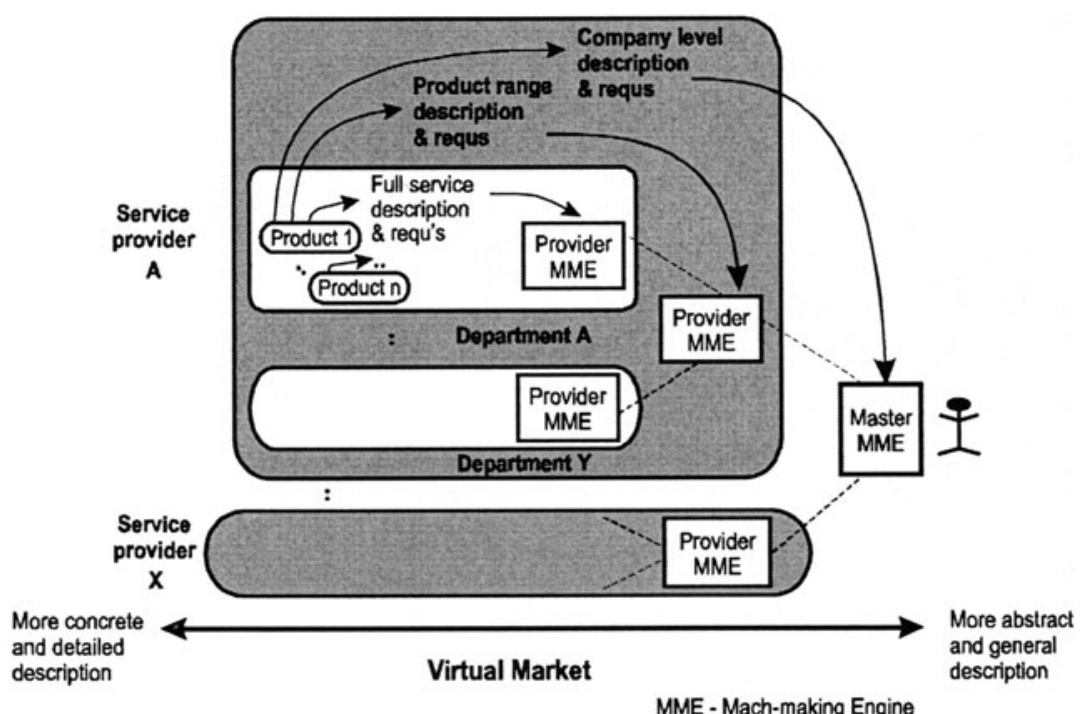

Figure 2. Mapping of staged advertising into a match-making architecture.

\subsection{Distributing the market place dialogue}

Having described how the market place information space can be distributed in 4.2, it is now possible to show how the dialogue between the customer and the market place will behave. In this scheme, the dialogue consists of a series of exchanges with different levels of MMEs (Figure 3). Every successful exchange with a level of MME will provide the customer with feedback about the level of the dialogue and the goods, which fit the search criteria. The response will also provide the form which the customer has to fill for the next phase of the dialogue and which will be directed to the next relevant level of MMEs.

Dialogue progress can now be regarded as successive exchanges of information going from the abstract to the specific, from the more common to the more private and from the general to the more sensitive as described in 4.1. This scheme can be used to build trust between the customer and the provider by providing intermediate forms of feedback, indicating whether the dialogue is heading in the right direction. Another advantage of the phased dialogue approach is that the client does not have to possess full knowledge and understanding of the market goods at the start of the dialogue because of the staged manner of the dialogue and the gradual increase in detail and complexity. 


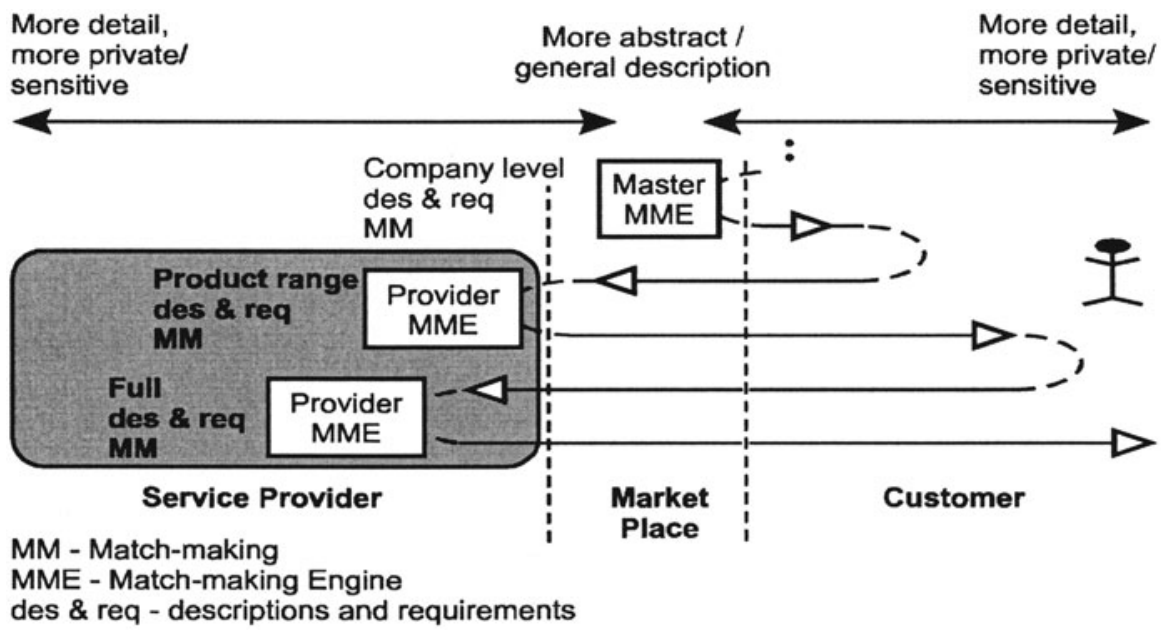

Figure 3. Phased dialogue implementation with a single provider.

In fact, it is also possible to employ the distributed market place in another manner, by allowing several dialogues to be conducted with multiple providers simultaneously. This, however, raises the problem of synchronising and managing these multiple dialogues, as well as the problem of agreement on common dialogues between the market place providers. This is further discussed in 5.2.4.

\section{CHECKING THE REMAINING REQUIREMENTS}

The distributed approach which purports to address the requirements stated in 2.2 and 2.3, must be validated against the requirements which were not considered of primary importance in 3.1 , in order to see whether the approach contradicts any of them, and if so, how the contradictions can be solved.

\subsection{Dealing with apparently contradictory requirements}

The following is a discussion of the requirements, which appear to contradict the distributed approach.

5.1.1 Create an audited and regulated market place. The requirement for an audited and regulated market place appears to favour a non-distributed market place, as the centralisation of the dialogue makes monitoring and control easier, but at the expense of the scaling characteristics of the market place. This however, requires the centralised market place to be a trusted entity.

Market places have a single (logical) point of entry for customers which can be replicated for performance and availability reasons. Thus, the initial stage of the dialogue always takes place at the market point of entry. However, the distribution of the market place as suggested by our approach, offers the option of centralised or distributed control of the subsequent dialogue phases.

The centralised dialogue directs all the interactions between the customer and the market place through the point of entry, thereby separating the customer from the 
providers and vice versa. Such a centralised approach provides a point of monitoring and control, which can be used for market regulation, as a point to inject market wide policy, as a point of auditing or market statistics collection. The distributed dialogue control starts off in a similar manner to the centralised one but then relinquishes control over the interactions, so that the customer can directly access the rest of the market place and the providers systems.

In the distributed approach the client is then free to interact with the service provider MMEs directly without the intervention of any intermediaries. The distributed approach scales better than the centralised approach as there is no centralised point of dialogue control (except of course at the client's end point), but leaves the responsibility of managing the dialogue with the client. This may force the client side to deal with issues such as asynchronous dialogues, monitoring and audit trail provision, separation of client from provider, reliability, security etc. This is likely to make the client side software larger and more complex. In the case of Web applet based solutions this is likely to make the downloading time of the client side applet unacceptable.

The centralised dialogue control approach appears not to scale, as it constitutes a bottleneck. However, by creating an individual session agent for each customer dialogue with the market place, and by distributing the load of such agents over a number of hosts, the problem of the bottleneck can be solved. This is further explained in Chapter 6.

5.1.2. Provide complete and dynamic control of the advertising. Distributing the information, which describes market place goods between and within the provider and the mediator, raises the problem of updating this information when goods change or are to be withdrawn from the market place. By using advanced distribution technology such as a CORBA compliant platform [11] or Java RMI [1], and designing the MMEs in which this information resides to provide the appropriate management functions, the complexity and overhead of updating the information will be outweighed by the advantages of the distributed approach.

5.1.3. Protect commercially sensitive information and business processes. Distributing the information about goods in the market place appears to raise the problem of letting business sensitive information leak outside the provider organisation. However, if sensitive information is kept within the provider organisation and accessed by the relevant MME only when the need arises, this danger is minimised.

\subsection{Requirements which are independent of the distributed approach}

The following requirements, which were considered to have a low priority in 3.1 are simplified or addressed by the distribution of the market place.

5.2.1. Bi-directional information exchange and symmetric match-making. Much of the focus of current electronic market places concerns the ability of customers to select, from a large information space, those providers' offers, which match their needs and desires. One of the requirements not addressed by the distribution aspects concerns the complementary need of providers to distinguish between different types of customers, enabling the targeting of specific goods to specific customers. This 
will help avoid unnecessary interaction with customers for whom certain types of goods cannot be offered, thus satisfying one of the provider's requirements listed in 2.3. This requires a bi-directional and symmetric exchange of information - not just in terms of offering choice by one side and making the selection by the other, but in the sense that both sides will have to provide information about what they guarantee to deliver and what they require the other party to provide. The bi-directional information exchange in a dialogue necessitates a symmetric match-making process, comparing the requirements of one party with what the other offers, and vice versa (Figure 4). The topic of symmetric match-making and its implementation in the ViMP prototype (6) is covered in [7] and [5].

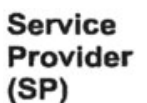

(SP)

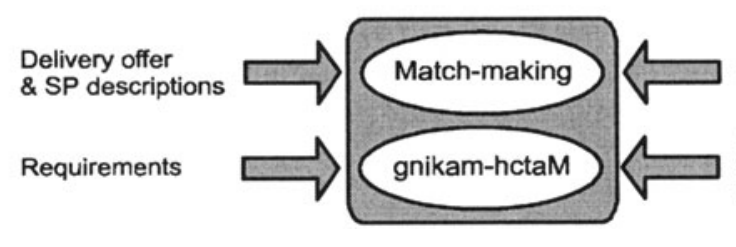

\section{Customer}

Requirements

Delivery offer

\& Customer descriptions

Figure 4. The symmetric match-making process in a virtual market place dialogue.

5.2.2. Protection from direct access. The customer session agent described in 5.1 .1 can also be used to protect the providers from direct access by the customer and likewise to protect the customer from direct contact with the providers. This may prevent failures arising from improper access to the providers, and prevent the providers from bombarding the customer with junk mail.

5.2.3 Interruptible long-life dialogues (asynchronous sessions). The customer session agent described in 5.1.1 can also be used to provide some level of persistence, thereby enabling the resumption of the dialogue in case of failure or when the customer wishes to interrupt the dialogue and resume it at a later point.

5.2.4. Supply necessary information only once ('One to many' dialogues). So far, the dialogue was discussed in terms of an exchange between a customer and a single provider. Where multiple service providers who offer similar goods come together and agree to form an electronic market place, several opportunities and problems arise. The inherent parallelism in such a market place, coupled with sufficient agreement or standardisation concerning the dialogue, makes it possible to conduct multiple simultaneous dialogues with multiple providers on behalf of each single customer (without having to repeat the same sequence of questions and answers for each service provider separately e.g. fill multiple forms).

The inherent parallelism in a distributed market place makes it possible to conduct multiple simultaneous dialogues with multiple providers on behalf of each single customer. However, 'one to many' dialogues raise two major problems, which must be addressed: 
- The extent to which agreement concerning the structure of the dialogue and the content of each stage of the dialogue is necessary between the market place participants [4]. However, this issue is outside the scope of this paper.

- The problem of synchronisation of the multiple interactions with different providers and the need to collate the replies and present them to the customer. A number of different approaches are possible but are outside the scope of this paper.

\section{THE VIMP PROTOTYPE IMPLEMENTATION}

The virtual market place architecture described in this paper has been implemented using Java (JDK 1.1.5) [1] on a CORBA [11] compliant platform (IONA's OrbixWeb 2.0.1 [9]). The prototype is called ViMP (Virtual Market Place) and has been working now for over a year, successfully animating electronic market place scenarios for car and fire insurance products. One of the key features of ViMP is that its major components are not market specific. Making the components data-driven so that any market specific information can be fed into them ensures their re-usability in different market places.

The current implementation of the Match-making Engine (MME) introduced in 4.2 is ViMP's modified CORBA Trading service [12], which supports several crucial extensions. Among them the symmetric match-making process described in 5.2.1 and in [7] and [5], as well as an advanced form of property and constraint language. The MMEs in the implementation described below are referred to as the Master Extended Trader (MET) - the market place point of entry, and the Provider Extended Trader (PET) - one or more of these reside in each provider organisation.

The description of ViMP in this chapter is divided into two parts: the market place set-up and management stage, and the customer-market place dialogue session. (Due to space limitations the main emphasis in this paper is on the latter).

\subsection{Market place set-up and management stage}

The market place set-up and management tools are primarily concerned with the view that the service providers have of the market place and how they control their input to the market. The tools also include facilities for on-going management of the market place by its managers (Figure 5).

The first stage in setting up the market place as far as a service provider is concerned, involves the definition of a product in accordance with the scheme described in 4.1. This is done by defining the goods features and requirements with a Product Manager. Once the product has been defined it can be advertised in the local Provider MME(s) and the Master MME by using the Offer manager. Two MME or extended trader layers exist in the current implementation, but more layers can be used if necessary.

One of the important issues that are tackled by our design is concerned with the link between the PETs, which reside within the organisational boundary, and the back office systems and dynamic property evaluators. A Dynamic Property Evaluator can be used to evaluate on-the-fly some product properties which have been previously advertised in the MME but whose value should be computed at 
search rather than at advertising time. This can be used, for example, in the premium calculation of an insurance product.

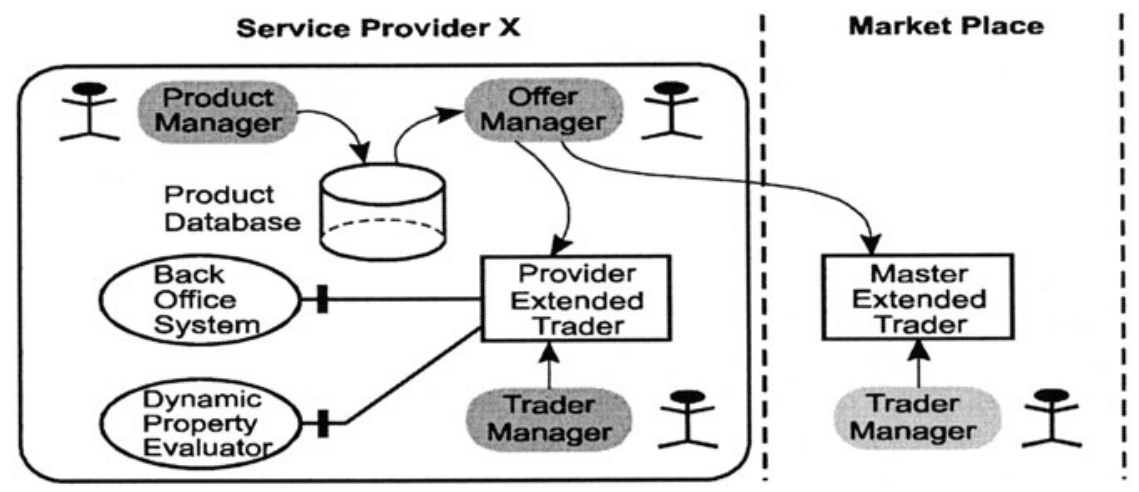

Figure 5. Provider-related ViMP components for market place set-up.

The offers advertised in the MMEs can be monitored and controlled using the Trader Manager inspection tool.

\subsection{Customer-market place dialogue session}

The dialogue session components and tools are primarily concerned with facilitating the interaction between the customer and the market place. A typical client session is shown in Figure 6.

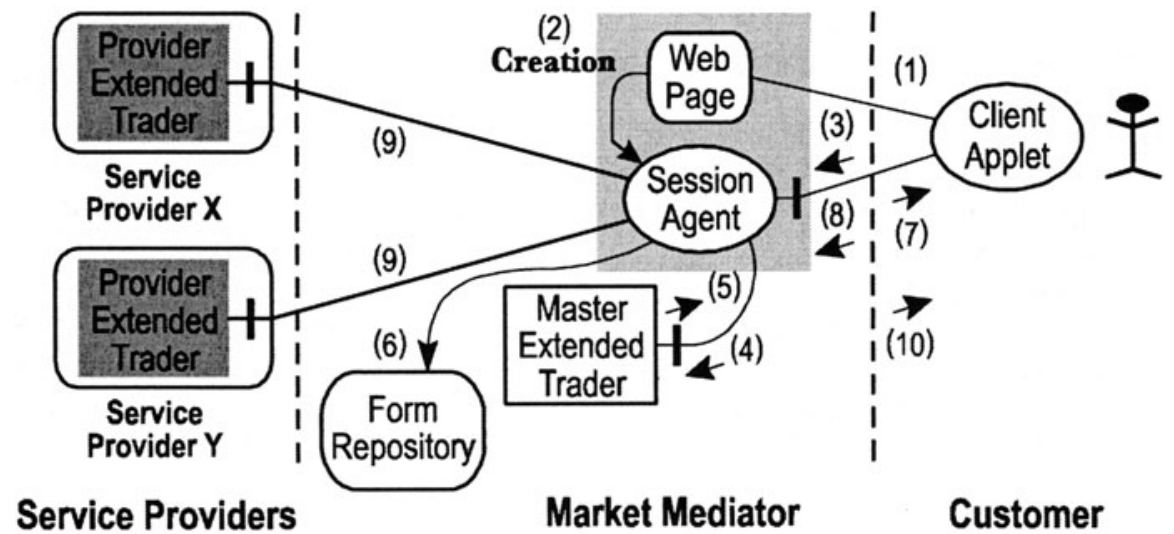

Figure 6. Schematic representation of a typical ViMP client session. 
Once the market place has been set up and the relevant products have been advertised, a customer can access the virtual market place through a web browser (1). The interaction with the market place is driven by a client applet that is downloaded from the ViMP web server. The web server spawns a Session Agent (2). The Session Agent (SA) represents the customer in the market place and is responsible for all the subsequent interactions between the customer and the market. The SA knows where to get the form for the next level of dialogue from, where to send the filled forms to, and how to collate the replies and present them to the customer.

The client applet represents the initial form, which has to be filled by the customer when initiating a query operation on the MET (3). The Session Agent directs the first filled form to the MET that searches through its database for previously posted service descriptions (4). If any matching offers are found, the MET returns a list of service specifications which consist of a high-level description of products which includes the name of the respective service provider (5).

The client can now select the providers he wishes to interact with. This selection is sent to the SA to request the relevant forms for the next level of the dialogue (6). The SA sends the requests to the corresponding Form Repositories and collates the results into a single form, which is sent back to the client (7). If this form is filled and returned to the SA (8), it forwards the appropriate information as query requests to the relevant Provider Extended Traders (9). Each reply, representing a successful match is displayed to the client (10). These can be either final service offers or another set of service descriptions in which case the selection procedure has to be repeated and another dialogue level is entered. Final service offers can be compared using the ViMP catalogue [15], purchased, and the necessary payment related activities will ensue.

We are now working on an enhanced version of ViMP in collaboration with a major financial service customer, with a view to deploying the system in a 'real' virtual market place.

\section{CONCLUSIONS AND FUTURE DIRECTIONS}

By carefully separating certain virtual market place aspects and by physically distributing them, it is possible to derive a market place architecture, which satisfies the important requirements of both the customers and providers of such markets. However, it is important to bear in mind that centralisation has advantages, and that distribution does not come for free - there is a price to pay in terms of the overall complexity of such market places. This complexity, however, is heavily outweighed by the advantages accrued by distribution.

Any virtual market place designer will be faced with the problem of deciding:

- How to separate and distribute the market place information space between the providers and the market place mediator?

- How to separate and distribute the phases of the dialogue to make the phases meaningful without overwhelming the customer with the volume of information given to and required from them? 
- How to productively exploit existing systems within the provider organisations, without blocking opportunities for change in the future?

Our architecture does not provide a single solution, which would fit every type of market place. Nor does the proposed architecture dictate exactly how to separate the issues which need separating and how to distribute them; this is highly dependent on the type of market place being dealt with and its players. The distributed virtual market place architecture points out how large the design space of such markets is in fact, giving but a glimpse of the vast spectrum of options facing the designer, the kind of trade-off between the options and the possible implications of their choices.

However, as far as virtual market places are concerned, these are early days many different market places will have to be studied before detailed and constructive advice can be given with any degree of confidence. This is where ViMP can help the ViMP architecture is accompanied by an integrated set of generic components and tools which have been designed and developed as a re-usable framework in which domain specific knowledge can be injected to suit different market requirements and circumstances. The market place components and tools enable:

- Creating a virtual market infrastructure by setting-up the components inside as well as outside the service providing companies.

- Describing and subsequently advertising goods into the market place.

- The client to search the market place.

- Conducting a multi-stage dialogue with multiple service providers simultaneously.

- Maintaining, updating and modifying service offers by the service providers.

- Managing and maintaining the entire market place.

The ViMP set of components and tools allows experimenting with different market place designs and implementations in a relatively straight-forward manner, without having to start from scratch every time.

There are still a number of areas which were not addressed in this paper and which require extensive research and development. Among these are security and fault tolerance. In addition, applying the virtual market place system to more serviceoriented (as opposed to product-based) markets is likely to lead to the identification of further system requirements.

\section{References}

[1] ARnold K, Gosling J., The Java Programming Language. Addison-Wesley, 1996, ISBN 0-201-63455-4.

[2] BAKOS Y., The emerging role of electronic marketplaces on the Internet, Communications of the ACM), pages 35-42, Vol. 41, No. 8 (Aug.) 1998. 
[3] Boll S., Gruener A., HaAf A. AND Klas W., EMP - A Database-Driven Electronic Market Place for Business-to-Business Commerce on the Internet, Journal of Distributed and Parallel Databases, 7, Kluwer Academic Publishers, Boston, 1999.

[4] Dogac A., Durusoy I., Arpinar S., Gokkoca E., Tatbul N. and Koksal P., METU_Emar: An Agent-Based Electronic Marketplace on the Web, Proceedings of the Second European Conference on Research and Advanced Technology for Digital Libraries, Eds. Nikolaou C. and Stephandis C. Lecture Notes in Computer Science 1513, Springer-Verlag, Berlin, 1998.

[5] Field S., Facciorusso C., Hoffner Y., Schade A. ANd Stolze M. Design Criteria for a Virtual Market Place (ViMP), Proceedings of the Second European Conference on Research and Advanced Technology for Digital Libraries, Eds. Nikolaou C. and Stephandis C. Lecture Notes in Computer Science 1513, Springer-Verlag, Berlin, 1998.

[6] Hamilton S. E-Commerce for the 21st Century, IEEE Computer, Vol. 30, No. 5, pp. 4447, May 1997.

[7] HofFNer, Y. AND SCHADE, A., Co-operation, Contracts, Contractual Match-Making and Binding, 2nd International Enterprise Distributed Object Computing Workshop (EDOC '98), 3-5 November 1998, San-Diego, USA.

[8] INSWEB CORPORATION, http://www.insweb.com

[9] Orbix: Programmer's Guide, Iona Technologies Ltd., Dublin, Republic of Ireland, 1993.

[10] Open Distributed Processing Reference Model, ISO/IEC 10476, ITU-T Recommendation X.900 (1995) Parts 1, 2, 3.

[11] The Common Object Request Broker: Architecture and Specification, Object Management Group, Revision 2.0, 1995.

[12] OMG, CORBA Trading Object Service, Document orbos/96-05-06, May 10, Object Management Group and X/Open, 1996.

[13] Quicken InsureMarket, http://www.insuremarket.com.

[14] Tenenbaum J.M., Chowdhry T.S. AND Hughes K. Eco System: An Internet Commerce Architecture, IEEE Computer, Vol. 30, No. 5, pp. 48-55, May 1997.

[15] Stolze, M. Soft Navigation in Product Catalogs, Proceedings of the Second European Conference on Research and Advanced Technology for Digital Libraries, Eds. Nikolaou C. and Stephandis C. Lecture Notes in Computer Science 1513, Springer-Verlag, Berlin, 1998.

\section{Biographies}

Christian Facciorusso, Simon Field, Yigal Hoffner and Andreas Schade are all members of the e-business Solutions Group.

Christian Facciorusso graduated in 1992 with a Diploma Degree in computer science from the University of Pisa in Italy. Simon Field is the manager of the ebusiness Solutions Group at IBM's Zurich Research Laboratory. Yigal Hoffner holds a BSc in Computer Science and Cybernetics and a PhD in Computer Science. Andreas Schade holds a diploma degree (Dipl.-Inf., 1994) and a doctoral degree (Dr. rer. nat., 1998) in computer science. 